\title{
BRCT Domain
}

National Cancer Institute

\section{Source}

National Cancer Institute. BRCT Domain. NCI Thesaurus. Code C14042.

BRCT domain was first identified in C-terminal domain of breast cancer associated protein and was found in many proteins that regulate the cellular response to DNA repair, where these domains act as multi-purpose protein-protein interaction modules. Crystallography study reveals that two $\sim 95$ amino acid BRCT repeats within the domain adopt similar structures that pack together in a head-to-tail arrangement. $(\mathrm{NCl})$ 